\title{
Retracted: A Study on Course of Infection and Haematological Changes in falciparum-Infected in Comparison with Artemisinin(s)-Treated Mice
}

\author{
Malaria Research and Treatment \\ Received 26 June 2014; Accepted 26 June 2014; Published 3 July 2014 \\ Copyright (C) 2014 Malaria Research and Treatment. This is an open access article distributed under the Creative Commons \\ Attribution License, which permits unrestricted use, distribution, and reproduction in any medium, provided the original work is \\ properly cited.
}

The article titled "A Study on Course of Infection and Haematological Changes in falciparum-Infected in Comparison with Artemisinin(s)-Treated Mice" [1], published in Malaria Research and Treatment, has been retracted as it was found to contain substantial flaws in its scientific content and experimental results.

\section{References}

[1] K. K. Kuthala, S. Meka, and S. Kanikaram, "A study on course of infection and haematological changes in falciparum-infected in comparis on with Artemisinin(s)-treated mice," Malaria Research and Treatment, vol. 2013, Article ID 426040, 10 pages, 2013. 


\title{
A Study on Course of Infection and Haematological Changes in falciparum-Infected in Comparison with Artemisinin(s)-Treated Mice
}

\author{
Kalyan Kumar Kuthala, Sowjanya Meka, and Sunita Kanikaram \\ Department of Zoology and Aquaculture, Acharya Nagarjuna University, Nagarjunanagar, Guntur, Andhra Pradesh 522 510, India
}

Correspondence should be addressed to Sunita Kanikaram; sunitamichael@yahoo.com

Received 29 December 2012; Revised 12 May 2013; Accepted 17 May 2013

Academic Editor: Mats Wahlgren

Copyright (c) 2013 Kalyan Kumar Kuthala et al. This is an open access article distributed under the Creative Commons Attribution License, which permits unrestricted use, distribution, and reproduction in any medium, provided the original work is properly cited.

To find out the efficacy and effect of artemisinin derivatives on haematological indices, C57BL/6J mice were challenged with Plasmodium falciparum and treated with therapeutic doses of AS, AE, and AL. Course of infection was studied in the infected and treated groups up to day 42. Peak level of parasitaemia (38\%) was observed on day 11 in infected group. Haematological indices indicated significant $(P<0.05)$ decrease in RBC, WBC, haemoglobin, packed cell volume, mean cell volume, and platelet counts in infected mice. But all the parameters were restored to normal values, and significant $(P<0.05)$ changes were observed in all drug-treated groups. Insignificant changes were observed for MCHC $(P>0.05)$ in all drug-treated groups. Percent of peak parasitaemia was much reduced in AL- (3.2\% on day 3$)$ treated group in comparison with AE- (2.4\% on day 4$)$ and AS- (4\% on day 2) treated groups. Parasites were completely cleared on day 6 in AS group, day 5 in AE group, and day 4 in AL group. Hence, our results strongly support that combination therapy has high efficacy rates than monotherapy. No adverse effects were observed on haematological parameters when animals were treated with therapeutic dosages.

\section{Introduction}

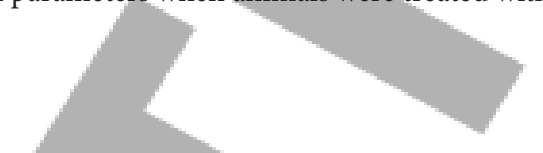

Despite advances in knowledge, malaria continues to cause significant morbidity and mortality worldwide. Over $40 \%$ of the world population lives in malaria-endemic areas and it is very high $(20 \%)$ in severe malaria (parasitaemia $>5 \%$ ). Today malaria is the most important problem for which an estimated 300-500 million cases were recorded and 1.52.7 million deaths occur each year [1]. Among them 19,500 death cases due to malaria have been recorded in India [2]. Mortality rate usually depends on the management of malaria which involves antimalarial drug resistance of Plasmodium falciparum and occurrence of systemic complications. Most of the systemic complications from malaria are mainly because of hyperparasitaemia [3].

Blood is the most easily accessible diagnostic tissue. Variations in haematological parameters are influenced by any disease condition which affects the haemopoietic physiology. This is likely to happen with an endemic disease such as malaria that affects the host homeostasis [4]. The target of malaria parasite is RBC so that peripheral blood smear examination is the major diagnostic tool of the disease. Microscopic diagnosis is the "imperfect gold standard" for malaria parasite detection and species identification. This technique requires technical expertise and time consuming in repeated smear examinations [5]. However, it is a valuable technique when performed correctly in the right hands but can be unreliable and perceived as useless when poorly executed $[6,7]$.

Haematological changes associated with malaria infection are well recognized, but specific changes may differ from the level of malaria endemicity, background haemoglobinopathy, nutritional status, demographic factors, and immunity to malaria [8]. Hence, haematological changes are the most common and important complications encountered and are considered a hallmark of malaria, playing a major role in the fatality. Prediction of the haematological changes enables the clinician to establish an effective and early therapeutic intervention in order to prevent the occurrence of 
major complications [3]. The haematological abnormalities that have been reported include anaemia, thrombocytopenia, lymphocytosis and rarely disseminated intravascular coagulation [9], leucopenia, leucocytosis, neutropenia, neutrophilia, and eosinophilia, and monocytosis also have been reported $[10,11]$.

Artemisinin(s) monotherapy and combination therapy (ACTs) are currently used as first line treatment for uncomplicated malaria. Artesunate is a semisynthetic derivative of artemisinin consisting of sodium succinyl salt of dihydroartemisinin, a potent blood schizonticide highly effective against multidrug resistant strains of $P$. falciparum. Hence it is widely used for the treatment of malaria [12]. $\alpha, \beta$ arteether (30:70 mixture of enantiomers) is a fast-acting blood schizonticide completed multicentric clinical trials in $P$. falciparum endemic areas and was found to be effective and marketed in 1997 as E Mal. Arteether has a higher safety margin compared to other artemisinin derivatives, has longer half-life, and produces high cure rates when administered in a short 3-dose (i.m.) regimen [13]. Artemether + lumefantrine combination combines the benefits of a rapid short-lived schizonticidal effect of arteether with a slower but longer acting schizonticidal effect of Lumefantrine, a highly lipophilic aryl amino alcohol.

The present study was aimed to reveal the effect of 3 frequently used artemisinin derivatives, namely, artesunate (oral), arteether (i.m.), and artemether + lumefantrine (oral) on haematological parameters. The present investigation was also aimed to study the course of infection in falciparuminfected and drug-treated mice to know the efficacy of Artemisinin derivatives based on parasite clearance time (PCT). Thus the aim of our study was to investigate the different hematological changes with $P$. falciparum malaria and to define the possible role of Plasmodium species in the pathogenesis related to haematological changes.

\section{Materials and Methods}

2.1. Animals. C57BL/6J male mice of age of 10 -week-old were purchased from National Centre for Laboratory Animal Sciences (N.C.L.A.S), National Institute of Nutrition (NIN), Hyderabad, India, and allowed to acclimatize for 15 days. Animals were fed with standard feed daily and water was given adlibitum at room temperature of $24 \pm 5^{\circ} \mathrm{C}$ with $12 \mathrm{hrs}$. light and dark cycle. The study was conducted in accordance with guidelines in the Guide of the Care and Use of Laboratory Animals [14].

2.2. Diagnosis, Collection, and Storage of Blood. For the present experiment the species of $P$. falciparum was collected from an infected person from Government General Hospital in Guntur, Andhra Pradesh. Patient is tested with SD BIOLINE Malaria rapid test (P.f/p.v) and blood smear examination by 10 to 15 years experienced microbiologist who confirmed $P$. falciparum with high parasitaemia. At the time of blood collection the patient does not receive any preantimalarial treatment even paracetamol. The blood containing $P$. falciparum was extracted from peripheral vein of hand of the infected individual using $5 \mathrm{~mL}$ sterile syringe.
Immediately blood was transferred to EDTA vacutainer $(\mathrm{BD}$ Franklin, USA); kept in thermocol ice box, and transferred to the laboratory. The obtained antigen was inoculated into the mice within half an hour of collection.

2.3. Preparation and Inoculation of Antigen. The collected blood was washed several times in phosphate buffered saline (PBS, pH 7.0) by centrifugation at $1000 \mathrm{rpm} / 15 \mathrm{~min}$. The washed erythrocytes were suspended in PBS and packed by centrifugation, and supernatant was removed by a pipette. The sediment with $P$. falciparum-infected erythrocytes was diluted with PBS. After obtaining P. $f$. antigen, the parasites were maintained experimentally in three C57BL/6J male mice, and the level of parasitaemia was monitored after the next day of inoculation by smear preparation. After the achievement of high level of parasitaemia (stock blood), blood samples were collected and diluted in normal saline at the ratio of $75 \%$ parasitized blood and $25 \%$ PBS. The diluted parasitized blood was then inoculated into different experimental mice groups on day "0" via intraperitoneal (i.p.) route because malaria parasite penetrates the peritoneal wall into the blood stream within one minute of inoculation. Mice of control group were inoculated with distilled water on day " 0 " and maintained as control.

2.4. Drug Administration. A total number of $40 \mathrm{C} 57 \mathrm{BL} / 6 \mathrm{~J}$ male mice were distributed into 5 groups, namely, control (CON), infected (INF), drug treated with artesunate (DT AS), drug treated with arteether (DT AE) and drug treated with artemether + lumefantrine (DT AL) of 8 animals each. For all drug-treated groups, therapeutic dosages according to $\mathrm{WHO}$ recommendation were administered. Doses were calculated according to the average body weight of the mice (approximately $30 \mathrm{gm} / \mathrm{mouse}$ ). For monotherapy, artesunate (AS) tablets (Falcigo) from Zydus Cadila Health Care Limited, India, and arteether (AE) (E Mal) from Themis Chemicals Limited, Mumbai, India, were obtained. For combination therapy, artemether + lumefantrine (AL) (Lumerax-20 DT) from Ipca Laboratories Limited, India, was obtained.

(i) Artesunate: through oral gavage in 4 (double divided dose), 2, 2, 2, and $2 \mathrm{mg} / \mathrm{kg}$ body weight for five days.

(ii) Arteether: intramuscularly in $3 \mathrm{mg} / \mathrm{kg}$ body weight for three days.

(iii) Artemether + Lumefantrine: through oral gavage in $3.5 \mathrm{mg} / \mathrm{kg}$ body weight for three days.

2.5. Course of Infection. Course of infection was studied in all the 12-week C57BL/6J experimental mice. Parasitaemia was monitored daily up to day " 42 " by making peripheral blood smears. Comparison of parasitaemia between infected mice and drug-treated mice revealed the efficacy of each drug used for the study. Thin blood films were made on the prelabelled slide with free flowing whole blood directly from the mouse tail snips after the first drop was wiped off with cotton wool. The blood films were stained with JSB I and JSB II for the detection of malarial parasites and for estimation of parasitaemia. After staining, slides were washed 
with tap water to remove excess stain and allowed to drain in a vertical position and to air dry. A field was selected where the RBCs are in an evenly distributed monolayer and observed under 100x oil immersion objective. A minimum of 1000 RBCs were counted from 10 fields under microscope, and the number of infected RBCs will be recorded. The percent of parasitaemia was determined by enumerating the number of infected RBCs per total number of RBCs counted (5):

$$
\text { parasitaemia } \%=\frac{\text { no. of infected RBCs }}{\text { no. of RBCs counted }} \times 100 \text {. }
$$

2.6. Evaluation of Responses. Parasite clearance time (PCT) was defined as the time from the start of treatment until first negative blood smear for asexual stages which remained negative for an additional 24 hours. For our study we followed 42-day followup for all treatment groups. Thus PCT was observed in all the experimental groups.

2.7. Estimation of Haematological Parameters. At the end of the series of experiments (i.e., after achieving first negative blood smear), 6 animals in all the drug-treated groups were sacrificed using chloroform anaesthesia. Six animals in infected and six animals in control group were sacrificed on day 28. Two animals in all the groups kept for 42 days, and one animal is used to calculate parasitaemia on each day. Blood samples were collected by cardiac puncture into EDTA vacutainer to see the frequency of haematological abnormalities especially anaemia, thrombocytopenia, and reduced blood counts in malaria. Whole blood was immediately analyzed for complete blood picture (CBP), that is, red blood cell (RBC) count, haemoglobin (HGB), hematocrit (HCT), mean cell volume (MCV), mean cell haemoglobin $(\mathrm{MCH})$, mean cell haemoglobin concentration (MCHC), white blood cell (WBC) count, and platelet (PLT) count using the fully automated ABX Pentra 60 + Analyser (Horiba ABX, Montpellier, France). Briefly, $53 \mu \mathrm{L}$ of blood was aspirated into a needle divided and distributed to the various chambers for sample analysis.

2.8. Statistical Analysis. The means, standard deviations of normally distributed data were compared between control versus infected group and infected versus drug-treated groups using Student's $t$-test with MINITAB 11.12.32. Bit statistical package and graphs were drawn from MS Excel 2010. The values were given as mean \pm SD and are statistically significant at $t>2.306, P<0.05^{*}$ (significant), $P<0.001^{* *}$ (more significant), and $P<0.0001^{* *}$ (highly significant). $P$ value more than 0.05 was considered as statistically not significant (NS).

\section{Results}

3.1. Observation of Plasmodium falciparum Erythrocytic Stages (Asexual Forms). In the present study, P. falciparum erythrocytic stages were developed in the blood of C57BL/6J mouse model after inoculation of $0.3 \mathrm{~mL}$. of $P . f$ antigen intramuscularly to the experimental mice. Various stages of parasite are seen in the blood films stained with JSB-I and JSB-II solutions. The blood films were scanned under high power objective $(\times 40)$ and examined closely under oil immersion objective $(\times 100)$. The various erythrocytic stages of $P$. falciparum were seen such as trophozoites (immature and mature), schizonts, and gametocyte. Appearance of ring forms at the edge of $\mathrm{RBC}$ is the characteristic feature of Plasmodium falciparum species (Figure 5).

The erythrocytic stages observed were as follows.

(1) Immature Trophozoite: undivided nucleated cells with blue-coloured cytoplasm or a ring of cytoplasm within the red cell.

(2) Mature Trophozoite: compact cytoplasm, enlarged amoeboid shape, and no ring structure.

(3) Schizont: individual nucleated cell distributed throughout the red cell in a circle.

3.2. Study of Course of Infection in Plasmodium falciparumInfected Mice. After inoculation of $P . f$. antigen on day " 0 " into the mice of infected (INF) group, the parasites started developing in the peripheral blood from day " 1 " onwards and reached to peak level on 11 th day with $38 \%$ parasitaemia. Then the parasitaemia gradually decreased and completely disappeared by day 28 (Figure 1).

3.3. Effect of Artesunate on Plasmodium falciparum in Experimental Mice. After inoculating P. $f$. antigen to mice of DT AS group, parasitic ring stags have appeared on 1st day. Then artesunate drug was administered orally for the next 5 days successively with $2 \mathrm{mg} / \mathrm{kg}$ body weight with a double divided dose on the first day. After treating the mice with drug, the peak level of infection was observed on day " 3 " with $3.5 \%$ of parasitaemia only. Then the parasitaemia gradually decreased and disappeared on the 6th day. But the blood smears were examined till the 42nd day and no parasites were observed (Figure 2). The Parasite Clearance Time (PCT) was 144 hours (6 days) with artesunate.

\subsection{Effect of Arteether on Plasmodium falciparum in Experi-} mental Mice. After inoculation of P. f. antigen to mice of DT AE group, parasitic ring stags have appeared on the 1st day. Arteether was administered (i.m.) on successive 3 days. After Arteether treatment, the peak level of infection was observed on day " 2 " with $4.2 \%$ parasitaemia. Then the parasites were completely disappeared by the 5 th day (Figure 3 ). The Parasite Clearance Time (PCT) was 120 hours (5 days) with arteether.

3.5. Effect of Artemether + Lumefantrine on Plasmodium falciparum in Experimental Mice. After giving P. $f$. antigen to the mice in DT AL group, parasitic ring stages have appeared on the 1st day. Then artemether + lumefantrine (artemisinin based combination therapy) was administered orally for the next three days. With the combination therapy, peak level of infection was observed on day " 3 " with $3.2 \%$ parasitemia, and no parasites were observed on day " 4 " till the 42 nd day (Figure 4). The Parasite Clearance Time (PCT) was 96 hours (4 days) with artemether + lumefantrine. 


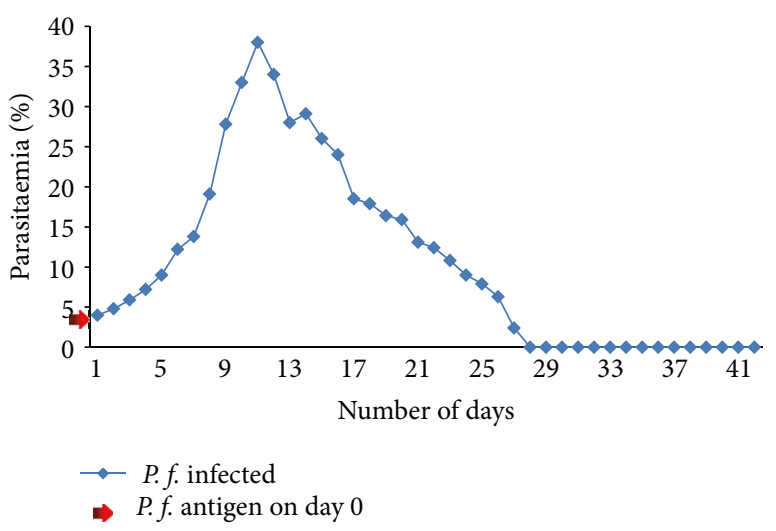

FIgure 1: Course of infection to Plasmodium falciparum in experimental C57BL/6J mice.

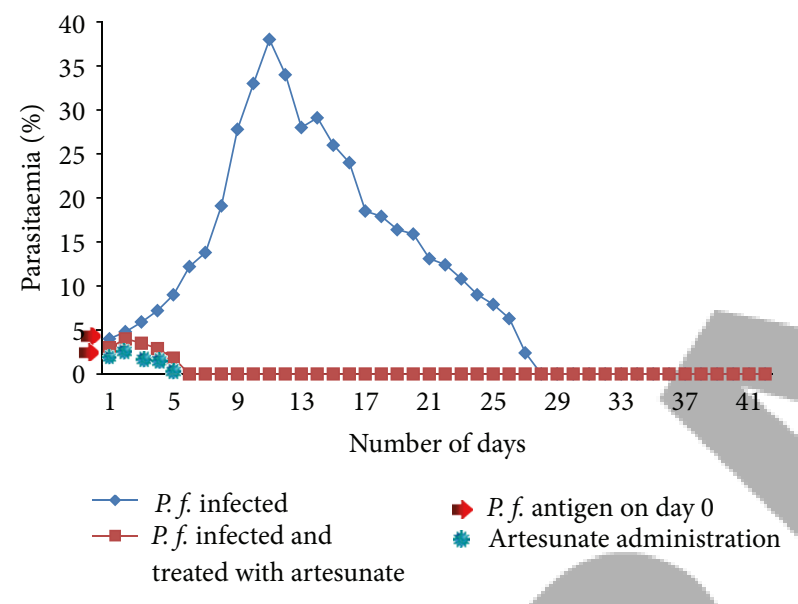

FIgURE 2: Effect of artesunate on Plasmodium falciparum in experimental C57BL/6J mice.

3.6. Changes in Haematological Values of Experimental C57BL/6J Mice due to Plasmodium falciparum. Table 1 shows changes in haematological values between control versus infected group. In the infected group the mean value for HGB is $7.93 \pm 0.175(P<0.0001), \mathrm{RBC}$ is $5.9 \pm 0.201(P<0.0001)$, $\mathrm{PCV}$ is $2892 \pm 1.46(P<0.0001), \mathrm{MCV}$ is $81.07 \pm 2.19$ $(<0.0001), \mathrm{MCH}$ is $27.67 \pm 1.17(0.0001), \mathrm{PLT}$ is $516 \pm 23.4$ $(<0.0001)$, WBC is $6957 \pm 102(<0.0001)$, neutrophils is $28.3 \pm$ $0.724(P<0.0001)$, lymphocytes is $69.70 \pm 2.00(P<0.0001)$, and eosinophils is $1.0 \pm 0.669(P<0.014)$. And these values were significantly decreased when compared to the control values $(12.85 \pm 0.321,8.0 \pm 0.141,38.67 \pm 1.97,91.48 \pm 1.64$, $32.33 \pm 1.26,685 \pm 20.7,9080 \pm 98.9,36.15 \pm 0.693,59.85 \pm 2.00$, and $2.0 \pm 0.187)$, respectively. $\mathrm{MCHC}(33.17 \pm 1.08, P>0.05)$ and monocytes $(1.0 \pm 0.190, P>0.05)$ have not shown significant change in the infected group when compared to control values $(34.42 \pm 1.12,1.0 \pm 0.228)$, respectively.

Table 2 shows changes in haematological values in infected (INF) versus artesunate drug-treated (DT AS) group. Significant changes were observed in $\operatorname{HGB}(P<0.0001)$, RBC $(P<0.0001)$, PCV $(P<0.01)$, MCV $(P<0.01)$, PLT $(P<$ $0.0001)$, WBC $(P<0.001)$, neutrophils $(P<0.0001)$, lym-

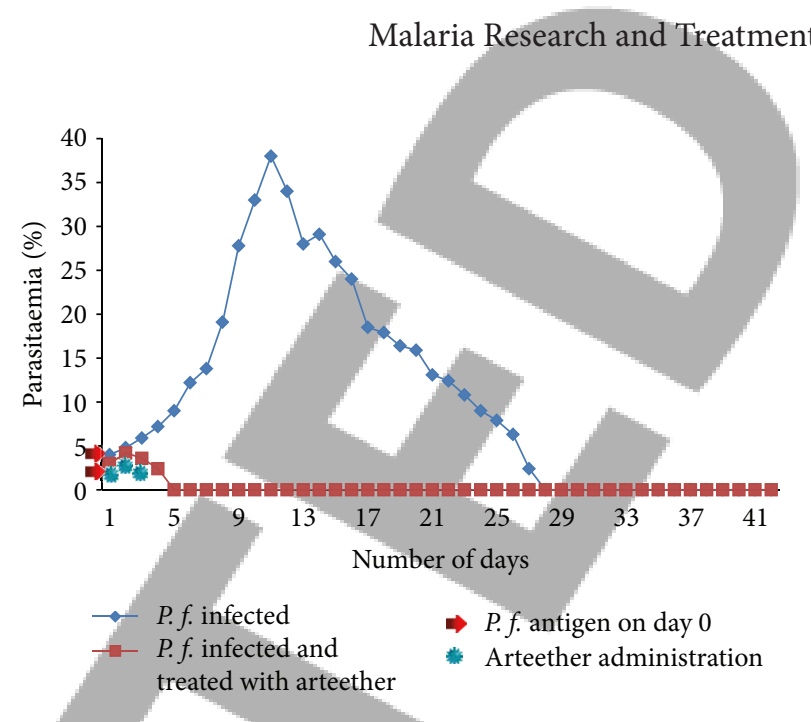

FIgURE 3: Effect of arteether on Plasmodium falciparum in experimental C57BL/6J mice.

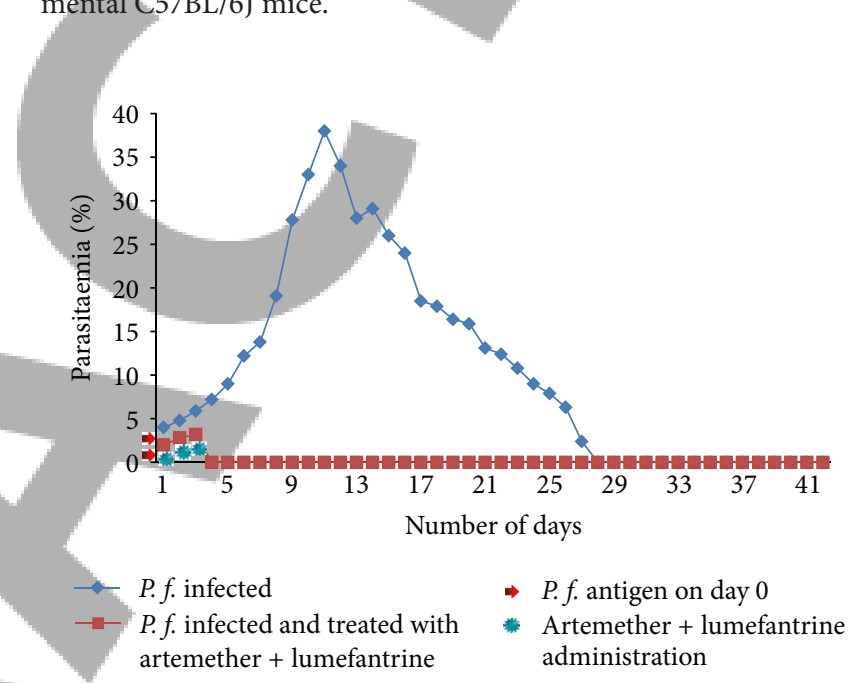

FIGURE 4: Effect of artemether + lumefantrine on Plasmodium falciparum in experimental C57BL/6J mice.

phocytes $(P<0.0001)$, and eosinophil $(P<0.05)$ in artesunate-treated group when compared with control. $\mathrm{MCH}$ $(P>0.05), \operatorname{MCHC}(P>0.05)$, and monocytes $(P>0.05)$ did not show statistically significant difference between INF and DT AS groups.

Table 3 shows changes in haematological values in infected (INF) versus arteether drug-treated (DT AE) group. HGB $(P<0.0001)$, RBC $(P<0.0001)$, PCV $(P<0.05)$, PLT $(P<0.0001)$, WBC $(P<0.001)$, neutrophils $(P<0.0001)$, lymphocytes $(P<0.0001)$, and eosinophil $(P<0.05)$ values were significantly increased in DT AE group when compared to INF group. MCV $(P>0.05), \mathrm{MCH}(P>0.05), \mathrm{MCHC}$ $(P>0.05)$, and monocytes $(P>0.05)$ have not shown significant change in DT AE group when compared to INF group.

Table 4 shows changes in haematological values in infected (INF) versus artemether + lumefantrine drug-treated (DT AL) group. HGB $(P<0.0001)$, RBC $(P<0.0001)$, PCV $(P<0.01)$, MCV $(P<0.001)$, PLT $(P<0.0001)$, WBC $(P<$ $0.001)$, neutrophils $(P<0.0001)$, lymphocytes $(P<0.01)$, 


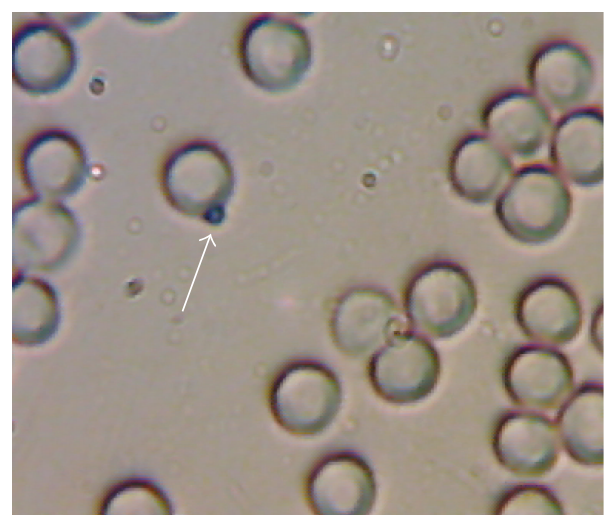

(a)

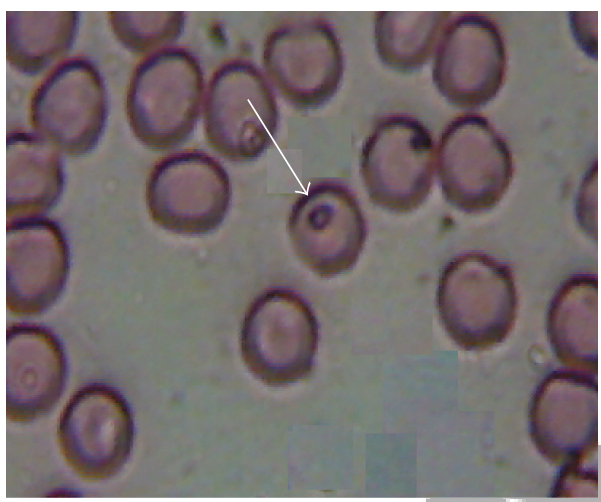

(c)

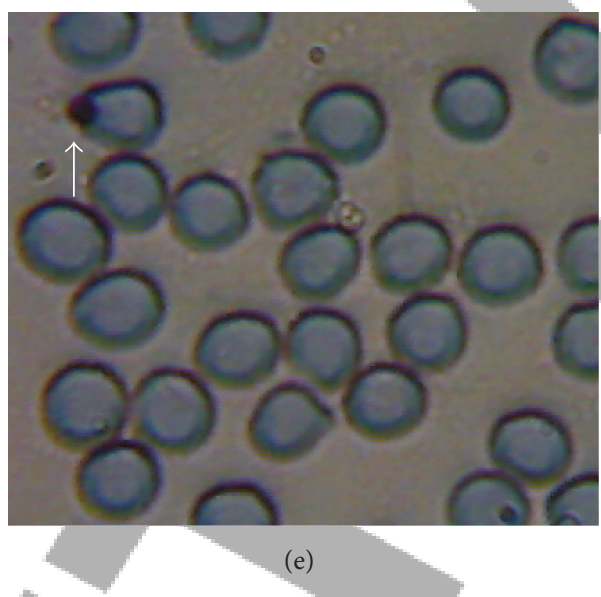

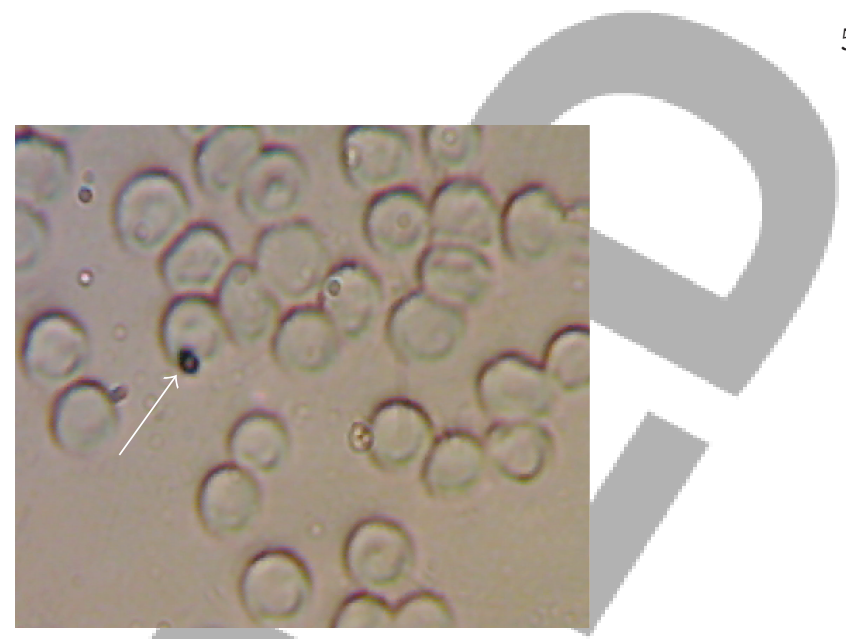

(b)

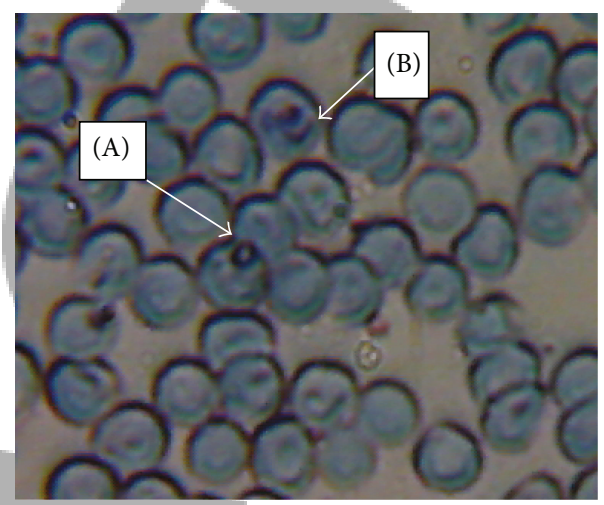

(d)

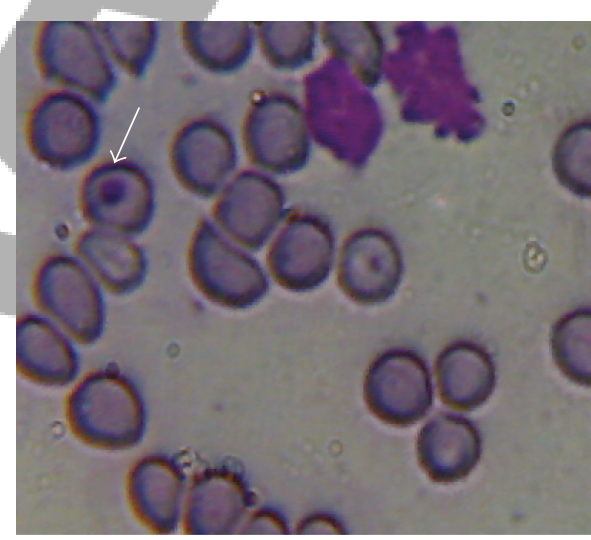

(f)

FIGURE 5: Developmental stages of Plasmodium falciparum in C57BL/6J mice blood. ((a) and (b)) Ring form or early trophozoite. ((c) and (d)-A) Developing trophozoite. (d)-B Early schizont. (e) Mature trophozoite showing clumped pigment. (f) Trophozoite.

and eosinophil $(P<0.05)$ in artemether + lumefantrinetreated group have significantly increased when compared to the infected group. Insignificant changes were observed for $\mathrm{MCH}(P>0.05)$, MCHC $(P>0.05)$, and monocytes $(P>0.05)$ between INF and DT AL groups.

\section{Discussion}

Severe falciparum malaria is associated with large parasite burdens. The peripheral blood parasite count is the prognostic indicator that correlates with the total parasite biomass 
TABLE 1: Changes in haematological values between control versus infected mice.

\begin{tabular}{|c|c|c|c|c|}
\hline \multirow[b]{2}{*}{ Parameter } & \multicolumn{2}{|c|}{ Experimental group $(N=6)$} & \multirow{2}{*}{$t$ value } & \multirow[b]{2}{*}{$P$ value } \\
\hline & $\begin{array}{c}\text { Control (CON) } \\
(\text { Day } 28)\end{array}$ & $\begin{array}{c}\text { Infected (INF) } \\
\quad(\text { Day 28) }\end{array}$ & & \\
\hline $\mathrm{HGB}(\mathrm{g} / \mathrm{dL})$ & $12.85 \pm 0.321$ & $7.93 \pm 0.175$ & 32.94 & $<0.0001^{* * *}$ \\
\hline $\operatorname{RBC}\left(10^{6} / \mathrm{mm}^{3}\right)$ & $8.0 \pm 0.141$ & $5.90 \pm 0.201$ & 20.84 & $<0.0001^{* * *}$ \\
\hline $\operatorname{PCV}(\%)$ & $38.67 \pm 1.97$ & $28.92 \pm 1.46$ & 9.74 & $<0.0001^{* * *}$ \\
\hline $\mathrm{MCV}(\mathrm{fL})$ & $91.48 \pm 1.64$ & $81.07 \pm 2.19$ & 9.34 & $<0.0001^{* * *}$ \\
\hline $\mathrm{MCH}(\mathrm{pg})$ & $32.33 \pm 1.26$ & $27.67 \pm 1.17$ & 6.65 & $<0.0001^{* * *}$ \\
\hline $\mathrm{MCHC}(\mathrm{g} / \mathrm{dL})$ & $34.42 \pm 1.12$ & $33.17 \pm 1.08$ & 1.96 & $>0.05^{\mathrm{NS}}$ \\
\hline $\operatorname{PLT}\left(10^{3} / \mathrm{mm}^{3}\right)$ & $685 \pm 20.7$ & $516.7 \pm 23.4$ & 13.19 & $<0.0001^{* * *}$ \\
\hline WBC (cells/mm³) & $9080 \pm 98.9$ & $6957 \pm 102$ & 36.61 & $<0.0001^{* * *}$ \\
\hline Neutrophils (\%) & $36.15 \pm 0.693$ & $28.3 \pm 0.724$ & 21.65 & $<0.0001^{* * *}$ \\
\hline Lymphocytes (\%) & $59.85 \pm 2.00$ & $69.70 \pm 2.00$ & 8.53 & $<0.0001^{* * *}$ \\
\hline Monocytes (\%) & $1.0 \pm 0.228$ & $1.0 \pm 0.117$ & 0.00 & $>0.05^{\mathrm{NS}}$ \\
\hline Eosinophils (\%) & $2.0 \pm 0.187$ & $1.0 \pm 0.669$ & 3.70 & $<0.05^{*}$ \\
\hline
\end{tabular}

The values are given as mean $\pm \mathrm{SD}$ are statistically significant at $t>2.306, P<0.05^{*}, P<0.001^{* *}$, and $P<0.0001^{* * *}$; not significant at $P>0.05$; HGB: hemoglobin; RBC: red blood cells; PCV: packed cell volume; MCV: mean cell volume; MCH: mean cell hemoglobin; MCHC: mean cell hemoglobin concentration; PLT: platelets; WBC: white blood cells.

TABLE 2: Changes in haematological values between infected versus artesunate drug-treated mice.

\begin{tabular}{|c|c|c|c|c|}
\hline \multirow{3}{*}{ Parameter } & & ntal group $(N=6)$ & \multirow{3}{*}{$t$ value } & \multirow{3}{*}{$P$ value } \\
\hline & \multicolumn{2}{|c|}{ (at the end of the experiment after parasite clearance) } & & \\
\hline & Infected (INF) & Drug-treated artesunate (DT AS) & & \\
\hline HGB (g/dL) & $7.93 \pm 0.175$ & $11.20 \pm 0.846$ & 9.28 & $<0.001^{* *}$ \\
\hline $\operatorname{RBC}\left(10^{6} / \mathrm{mm}^{3}\right)$ & $5.90 \pm 0.201$ & $7.95 \pm 0.0894$ & 22.73 & $<0.0001^{* * *}$ \\
\hline $\operatorname{PCV}(\%)$ & $28.92 \pm 1.46$ & $33.67 \pm 2.94$ & 3.54 & $<0.05^{*}$ \\
\hline MCV (fL) & $81.07 \pm 2.19$ & $86.83 \pm 3.19$ & 3.65 & $<0.05^{*}$ \\
\hline $\mathrm{MCH}(\mathrm{pg})$ & $27.67 \pm 1.17$ & $28.43 \pm 0.625$ & 1.42 & $>0.05^{\mathrm{NS}}$ \\
\hline $\mathrm{MCHC}(\mathrm{g} / \mathrm{dL})$ & $33.17 \pm 1.08$ & $32.75 \pm 1.73$ & 0.50 & $>0.05^{\mathrm{NS}}$ \\
\hline $\operatorname{PLT}\left(10^{3} / \mathrm{mm}^{3}\right)$ & $516.7 \pm 23.4$ & $675 \pm 11.0$ & 15.02 & $<0.0001^{* * *}$ \\
\hline $\mathrm{WBC}\left(\right.$ cells $\left./ \mathrm{mm}^{3}\right)$ & $6957 \pm 102$ & $8700 \pm 522$ & 8.04 & $<0.001^{* *}$ \\
\hline Neutrophils (\%) & $28.3 \pm 0.724$ & $40 \pm 0.754$ & 27.43 & $<0.0001^{* * *}$ \\
\hline Lymphocytes (\%) & $69.70 \pm 2.00$ & $57.67 \pm 2.80$ & 8.56 & $<0.0001^{* * *}$ \\
\hline Monocytes (\%) & $1.0 \pm 0.117$ & $1.0 \pm 0.126$ & 0.00 & $>0.05^{\mathrm{NS}}$ \\
\hline Eosinophils (\%) & $1.0 \pm 0.669$ & $2.0 \pm 0.518$ & 2.69 & $<0.05^{*}$ \\
\hline
\end{tabular}

The values are given as mean $\pm \mathrm{SD}$ are statistically significant at $t>2.306, P<0.05^{*}, P<0.001^{* *}$, and $P<0.0001^{* * *}$; not significant at $P>0.05$; HGB: hemoglobin; RBC: red blood cells; PCV: packed cell volume; MCV: mean cell volume; MCH: mean cell hemoglobin; MCHC: mean cell hemoglobin concentration; PLT: platelets; WBC: white blood cells.

control animals. The reduction was more evident in falciparum infection. It is thought to result from a combination of haemolysis of parasitized red blood cells, accelerated removal of both parasitized and innocently unparasitized red cells, depressed as well as ineffective erythropoiesis with dyserythropoietic changes, and anaemia of chronic disease [15]. Other factors contributing to anaemia in malaria include decreased red blood cell deformability, splenic phagocytosis, and/or pooling. So they have an increased rate of clearance from the circulation [16]. Malaria parasite within RBCs, ingest and digest haemoglobin more than it needs for its own metabolism. Destruction of RBC following parasitisation cannot account for the degree of anaemia observed during malaria infection, suggesting that the destruction of uninfected RBC ( $\mathrm{uRBC}$ ) is the major cause of haemoglobin (HGB) loss [17].

The low PCV value with a correspondent high density of malaria parasite was suggested to be due to excessive destruction of red blood cells by the malaria parasites. As noted in this study, it was established that the more malaria parasites in the blood circulation cause more destruction of the red blood cells as demonstrated by the low PCV. The low PCV may necessitate the need for blood transfusion which in turn has a high risk of transmitting viral hepatitis, HIV, 
TABLE 3: Changes in haematological values between infected versus arteether drug-treated mice.

\begin{tabular}{|c|c|c|c|c|}
\hline \multirow{3}{*}{ Parameter } & \multicolumn{2}{|c|}{ Experimental group $(N=6)$} & & \multirow{3}{*}{$P$ value } \\
\hline & \multicolumn{2}{|c|}{ (at the end of the experiment after parasite clearance) } & & \\
\hline & Infected (INF) & Drug-treated arteether (DT AE) & & \\
\hline $\mathrm{HGB}(\mathrm{g} / \mathrm{dL})$ & $7.93 \pm 0.175$ & $11.08 \pm 0.770$ & 29.53 & $<0.0001^{* * *}$ \\
\hline $\mathrm{RBC}\left(10^{6} / \mathrm{mm}^{3}\right)$ & $5.90 \pm 0.201$ & $7.73 \pm 0.154$ & 17.66 & $<0.0001^{* * *}$ \\
\hline PCV $(\%)$ & $28.92 \pm 1.46$ & $31.67 \pm 1.86$ & 2.84 & $<0.05^{*}$ \\
\hline MCV (fL) & $81.07 \pm 2.19$ & $86.17 \pm 5.49$ & 2.11 & $>0.05^{\mathrm{NS}}$ \\
\hline $\mathrm{MCH}(\mathrm{pg})$ & $27.67 \pm 1.17$ & $27.6 \pm 1.23$ & 0.10 & $>0.05^{\mathrm{NS}}$ \\
\hline $\mathrm{MCHC}(\mathrm{g} / \mathrm{dL})$ & $33.17 \pm 1.08$ & $33.35 \pm 0.985$ & 0.30 & $>0.05^{\mathrm{NS}}$ \\
\hline $\operatorname{PLT}\left(10^{3} / \mathrm{mm}^{3}\right)$ & $516.7 \pm 23.4$ & $650 \pm 22.8$ & 10.00 & $<0.0001^{* * *}$ \\
\hline $\mathrm{WBC}\left(\right.$ cells $\left./ \mathrm{mm}^{3}\right)$ & $6957 \pm 102$ & $8983 \pm 475$ & 10.22 & $<0.001^{* * *}$ \\
\hline Neutrophils (\%) & $28.3 \pm 0.724$ & $37.01 \pm 0.708$ & 21.08 & $<0.0001^{* * *}$ \\
\hline Lymphocytes (\%) & $69.70 \pm 2.00$ & $60.67 \pm 1.63$ & 8.57 & $<0.0001^{* * *}$ \\
\hline Monocytes (\%) & $1.0 \pm 0.117$ & $1.0 \pm 0.210$ & 8.57 & $>0.05^{\mathrm{NS}}$ \\
\hline Eosinophils (\%) & $1.0 \pm 0.669$ & $2.0 \pm 0.729$ & 8.57 & $<0.05^{*}$ \\
\hline
\end{tabular}

The values are given as mean \pm SD are statistically significant at $t>2.306, P<0.05^{*}, P<0.001^{* *}$, and $P<0.0001^{* * *}$; not significant at $P>0.05$; HGB: hemoglobin; RBC: red blood cells; PCV: packed cell volume; MCV: mean cell volume; MCH: mean cell hemoglobin; MCHC: mean cell hemoglobin concentration; PLT: platelets; WBC: white blood cells.

TABLE 4: Changes in haematological values between infected versus artemether-lumefantrine drug-treated mice.

\begin{tabular}{|c|c|c|c|c|}
\hline \multirow[t]{2}{*}{ Parameter } & & $\begin{array}{l}\text { xperimental group }(N=6) \\
\text { the end of the experiment })\end{array}$ & \multirow[t]{2}{*}{$t$ value } & \multirow[t]{2}{*}{$P$ value } \\
\hline & Infected (INF) & Drug-treated artemether-lumefantrine (DT AL) & & \\
\hline HGB (g/dL) & $7.93 \pm 0.175$ & $11.08 \pm 0.770$ & 9.77 & $<0.001^{* *}$ \\
\hline $\operatorname{RBC}\left(10^{6} / \mathrm{mm}^{3}\right)$ & $5.90 \pm 0.201$ & $7.78 \pm 0.317$ & 12.23 & $<0.0001^{* * *}$ \\
\hline PCV (\%) & $28.92 \pm 1.46$ & $33.67 \pm 2.94$ & 3.54 & $<0.05^{*}$ \\
\hline $\mathrm{MCV}(\mathrm{fL})$ & $81.07 \pm 2.19$ & $87.0 \pm 1.79$ & 5.14 & $<0.001^{* *}$ \\
\hline $\mathrm{MCH}(\mathrm{pg})$ & $27.67 \pm 1.17$ & $28.7 \pm 1.16$ & 1.54 & $>0.05^{\mathrm{NS}}$ \\
\hline $\mathrm{MCHC}(\mathrm{g} / \mathrm{dL})$ & $33.17 \pm 1.08$ & $33.33 \pm 1.34$ & 1.54 & $>0.05^{\mathrm{NS}}$ \\
\hline $\operatorname{PLT}\left(10^{3} / \mathrm{mm}^{3}\right)$ & $516.7 \pm 23.4$ & $680 \pm 11.4$ & 15.38 & $<0.0001^{* * *}$ \\
\hline $\mathrm{WBC}\left(\right.$ cells $\left./ \mathrm{mm}^{3}\right)$ & $6957 \pm 102$ & $8033 \pm 378$ & 6.74 & $<0.001^{* *}$ \\
\hline Neutrophils (\%) & $28.3 \pm 0.724$ & $41.00 \pm 1.41$ & 19.58 & $<0.0001^{* * *}$ \\
\hline Lymphocytes (\%) & $69.70 \pm 2.00$ & $58.33 \pm 4.55$ & 5.61 & $<0.001^{* *}$ \\
\hline Monocytes (\%) & $1.0 \pm 0.117$ & $1.0 \pm 0.297$ & 0.00 & $>0.05^{\mathrm{NS}}$ \\
\hline Eosinophils (\%) & $1.0 \pm 0.669$ & $2.0 \pm 0.566$ & 0.00 & $<0.05^{*}$ \\
\hline
\end{tabular}

The values are given as mean $\pm \mathrm{SD}$ are statistically significant at $t>2.306, P<0.05^{*}, P<0.001^{* *}$, and $P<0.0001^{* * *}$; not significant at $P>0.05$; HGB: hemoglobin; RBC: red blood cells; PCV: packed cell volume; MCV: mean cell volume; MCH: mean cell hemoglobin; MCHC: mean cell hemoglobin concentration; PLT: platelets; WBC: white blood cells.

and other associated risks. The significant reduction in PCV level indicates a relationship between malaria parasite and anaemia [18].

Statistically insignificant changes were observed in $\operatorname{MCHC}(P>0.05)$ and monocyte $(P>0.05)$ values in infected mice when compared to control mice. MCHC levels were not significantly changed which is consistent with the earlier reports [19]. In our study we observed that there was a significant reduction in total WBC counts $(P<0.0001)$. Leucopenia appears to be a common finding in both nonimmune patients with falciparum malaria and semi-immune children living in malaria-endemic regions [20]. The differential leucocyte count showed normal monocytic, eosinophilic counts and decreased neutrophilic counts.
Similarly in the majority of cases, either neutropenia or neutrophilia was reported [20]. Phagocytosis of malaria pigment is by monocyte/macrophages and less frequently by neutrophils $[15,20,21]$. Monocytes and rarely neutrophils contained malaria pigment and in very rare cases, erythrophagocytosis by monocytes was also observed in some studies. Our findings also showed that artemisinin(s) given at the therapeutic doses may not cause neutropenia, which is consistent with prior studies [22, 23].

Lymphocytes, particularly $\mathrm{T}$ cells, play a major role in immunity to falciparum malaria by releasing proinflammatory cytokines. However excessive secretion of proinflammatory cytokines has been shown to contribute to the severity in humans [24-26]. Our study showed that mice infected with 
falciparum had a higher lymphocytic count, and this may represent overstimulation of the proinflammatory pathway. Further studies on the role of lymphocytes are required to determine the significance of our findings.

It is a general consensus that thrombocytopenia is very common in falciparum malaria $[27,28]$ and usually believed that a significant reduction in platelet counts $(P<0.0001)$ than control animals. Thrombocytopenia seems to be due mainly to a reduced platelet life span and splenic pooling. The reduced platelet life span may be caused by binding of malaria antigen onto platelets followed by antibody-mediated phagocytosis [29] or to platelet activation in vivo. Macrophage activation and hyperplasia especially in the spleen may also play a role [30]. The release of platelet contents can activate the coagulation cascade and contributes to decreased inhibitors concentration and consequently further thrombocytopenia [31].

Artemisinin derivatives are most effective against Plasmodium parasite (as monotherapies); combination therapies consisting of artemisinin(s) and other antimalarial drugs have been demonstrated to have better parasite clearance and efficacies [32-34]. Antimalarial treatment with artemisinin or one of its derivatives is associated with a more rapid decline in parasitaemia than with other antimalarial drugs [35]. Artemisinins induce a decrease in parasitized RBC deformability. In the presence of heme $\mathrm{Fe}^{++}$, these drugs generate carbon centered free radicals that could damage the RBC membrane or cytoskeleton and thereby increase the rigidity of the infected RBC. Artesunate, by acting on young ring forms, attenuated the reduction in deformability parasite, prevented their development to more rigid mature trophozoites, and thereby attenuated the reduction in deformability associated with continued parasite growth. Artesunate induces changes either in the parasite or in the RBC directly and led to increased antigenicity and thus increased opsonization. Terminal half-lives of the orally administered drugs are usually less than $2 \mathrm{~h}$. We observed that once daily administration with artemisinin derivatives provides equivalent cure rates to more frequent administration which is consistent with prior studies [36,37]. In artesunate group peak of parasitaemia on day 3 with $3.5 \%$ and parasites were completely cleared on day 6 . First negative blood smear observed on day 6 . The peak level of parasitaemia was reduced when compared to AE-treated group $(4.2 \%$ on day $2)$. Insignificant changes were observed in $\mathrm{MCH}(P>0.05)$, MCHC $(P>0.05)$, and monocytes $(P>0.05)$ for artesunatetreated group.

Alpha beta arteether is an ethyl derivative of artemisinin which is an efficient schizonticidal drug in mild malaria. The clinical efficacy of arteether is characterized by an almost immediate onset and rapid reduction in parasitaemia, with complete clearance in most cases within 48 hours. But in our study we observed delayed parasite clearance time in $\mathrm{AE}$-treated groups when compared to previous studies. In arteether group parasites cleared on day 5 . We observed positive blood smears for one additional day after the completion of 3-day drug course. The peak level of parasitaemia (4.2\% on day 2) was increased when compared to AS-treated group (4 on day 2). Insignificant changes were observed in MCV
$(P>0.05), \mathrm{MCH}(P>0.05), \operatorname{MCHC}(P>0.05)$, and monocytes $(P>0.05)$ for arteether-treated group. No adverse effects were observed on hematological parameters when animals-treated with $\alpha, \beta$ arteether which are consistent with prior studies $[38,39]$.

AE showed lower efficacy than AL and AS. The levels of efficacy are similar to the findings of the recent studies on ACTs in Africa which revealed that AL has higher efficacy rates when compared to other antimalarials [40-43]. In our study we observed rapid parasite clearance in artemether + lumefantrine-treated group in comparison with artesunateand arteether-treated animals which are in agreement with previous studies. Our results strongly support that combination therapies have high efficacy rates than monotherapy. Artemisinin(s) can be used alone, but this leads to high rate of recrudescence (return of parasites), and there is a possibility of emergence of resistant strains to the single drug treatment on repeated and inappropriate use and other drugs are required to all parasites and to prevent recrudescence [44]. But in our study no parasite recrudescence was observed in monotherapy and combination therapy. But there is a marked variation in peak level parasitaemia and clearance time. In AL group parasites cleared on day 4 . We observed positive blood smears for one additional day after the completion of 3-day drug course. The peak level of parasitaemia (3.2\% on day 3) was decreased when compared to AS- (4\%) and AE- (4.2\%) treated groups. The rate of parasite clearance was used as a measure of the artemisinin pharmacodynamic effect in vivo [35]. Artemisinin resistance is characterized by prolongation in clearance times [18]. There is clear evidence that combinations improve efficacy without increasing toxicity. We found higher efficacy rates when animals were treated with combination (AL) drug than monotherapy. We observed positive blood smears on day 3 for all treatment groups. When compared to AS and AE groups, AL group showed higher efficacy rates by clearing parasites on day 4 . Insignificant changes were observed in $\mathrm{MCHC}(P>0.05) 0$ and monocytes $(P>0.05)$ for artemether + lumefantrinetreated group.

\section{Conclusion}

Considering the higher efficacy rates of artemether +lumefantrine (AL) as compared with artesunate (AS) and arteether (AE), we conclude that AL is clinically more effective than AS and AE. No adverse effects were observed on haematological parameters when animals were treated with artemisinin derivatives. Artemisinin resistance is characterized by prolongation in clearance times which we observed in the present study. The result of falciparum positive blood smear in all treatment groups on day $3(72 \mathrm{~h})$ was a good predictor for treatment failure and considered as a simple screening measure for artemisinin resistance.

\section{Conflict of Interests}

The authors declare that they do not have conflict of interests. 


\section{Acknowledgments}

The authors are extremely thankful to the University Grants Commission, New Delhi, India, for providing financial assistance in the form of Major Research Project (2010-2013) to carry out this work. My Special thanks to Professor Y. Prameela Devi, Department of Zoology, Kakatiya University, Warangal, India, for helping me to write up the Major Research Project. Also thankful to Professor V. Viveka Vardhani (Former Head) and Dr. K. Veeraiah, Head of the Department of Zoology and Aquaculture, for providing facilities through UGC-SAP-DRS and their cooperation during the course of work.

\section{References}

[1] A. H. Abro, A. M. Ustadi, N. J. Younis, A. S. Abdou, D. Al Hamed, and A. A. Saleh, "Malaria and hematological changes," Pakistan Journal of Medical Sciences, vol. 24, no. 2, pp. 287-291, 2008.

[2] V. P. Sharma, "Roll back of malaria," Current Science, vol. 75, no. 8, pp. 756-757, 1998.

[3] K. Taha, S. Zein El-Dein, and M. Idrees, "Haematological changes in malaria: relation to Plasmodium species," Kuwait Medical Journal, vol. 39, no. 3, pp. 262-267, 2007.

[4] R. N. Maina, D. Walsh, C. Gaddy et al., "Impact of Plasmodium falciparum infection on haematological parameters in children living in Western Kenya," Malaria Journal, vol. 9, no. 3, supplement, article S4, 2010.

[5] "World Health Organization, New perspective, malaria diagnosis," Geneva, Switzerland, 2000.

[6] T. B. Lathia and R. Joshi, "Can hematological parameters discriminate malaria from nonmalarious acute febrile illness in the tropics?" Indian Journal of Medical Sciences, vol. 58, no. 6, pp. 239-244, 2004.

[7] P. C. Wever, Y. M. C. Henskens, P. A. Kager, J. Dankert, and T. Van Gool, "Detection of imported malaria with the Cell-Dyn 4000 hematology analyzer," Journal of Clinical Microbiology, vol. 40, no. 12, pp. 4729-4731, 2002.

[8] R. N. Price, J. A. Simpson, F. Nosten et al., "Factors contributing to anemia after uncomplicated falciparum malaria," American Journal of Tropical Medicine and Hygiene, vol. 65, no. 5, pp. 614622, 2001.

[9] C. A. Facer, "Haematological aspect of malaria," in Infection and Haematology, pp. 259-294, Oxford Butterworth Heineman Limited, 1994.

[10] G. S. Murphy and E. C. Oldfield, "Falciparum malaria," Infectious Disease Clinics of North America, vol. 10, no. 4, pp. 747-775, 1996.

[11] J. H. Jandle, "Hemolytic anaemias caused by infection of red blood cells," in Blood, pp. 473-501, Little brown company, New York, NY, USA, 2nd edition, 1996.

[12] M. A. Van Agtmael, S. Cheng-Qi, J. X. Qing, R. Mull, and C. J. Van Boxtel, "Multiple dose pharmacokinetics of artemether in Chinese patients with uncomplicated falciparum malaria," International Journal of Antimicrobial Agents, vol. 12, no. 2, pp. 151-158, 1999.

[13] G. P. Dutta and R. Tripathi, "New antimalarial drug development in India: arteether $\alpha, \beta$-a blood schizontocide," Proceedings of the Indian National Science Academy, vol. 69, no. 6, pp. 861-870, 2003.
[14] Guide for the Care and Use of Laboratory Animals, Institute of Laboratory Animal Resources Commission on Life Sciences, National Research Council, Washington, DC, USA, 1996.

[15] L. H. Perrin, L. J. Mackey, and P. A. Miescher, "The hematology of malaria in man," Seminars in Hematology, vol. 19, no. 2, pp. 70-82, 1982.

[16] A. M. Dondorp, B. J. Angus, K. Chotivanich et al., "Red blood cell deformability as a predictor of anemia in severe falciparum malaria," American Journal of Tropical Medicine and Hygiene, vol. 60, no. 5, pp. 733-737, 1999.

[17] H. Ekvall, "Malaria and anemia," Current Opinion in Hematology, vol. 10, no. 2, pp. 108-114, 2003.

[18] A. M. Dondorp, F. Nosten, P. Yi et al., "Artemisinin resistance in Plasmodium falciparum malaria," New England Journal of Medicine, vol. 361, no. 5, pp. 455-467, 2009.

[19] G. Inocent, N. Djuidje Marceline, M. J. Pankoui Bertrand, and K. Fotso Honore, "Iron status of malaria patients in DoualaCameron," Pakistan Journal of Nutrition, vol. 7, no. 5, pp. 620624, 2008.

[20] S. H. Abdalla, "Peripheral blood and bone marrow leucocytes in Gambian children with malaria: numerical changes and evaluation of phagocytosis," Annals of Tropical Paediatrics, vol. 8, no. 4, pp. 250-258, 1988.

[21] O. K. Amodu, A. A. Adeyemo, P. E. Olumese, and R. A. Gbadegesin, "Intraleucocytic malaria pigment and clinical severity of malaria in children," Transactions of the Royal Society of Tropical Medicine and Hygiene, vol. 92, no. 1, pp. 54-56, 1998.

[22] J. S. Aprioku and A. W. Obianime, "Biochemical, haematological and reproductive indices in some biochemical systems," Insight Pharmaceutical Sciences, vol. 1, no. 1, pp. 1-10, 2011.

[23] C. Maiteki-Sebuguzi, P. Jagannathan, V. M. Yau et al., "Safety and tolerability of combination antimalarial therapies for uncomplicated falciparum malaria in Ugandan children," Malaria Journal, vol. 7, article 106, 2008.

[24] M. Ho, T. Schollaardt, S. Snape, S. Looareesuwan, P. Suntharasamai, and N. J. White, "Endogenous interleukin-10 modulates proinflammatory response in Plasmodium falciparum malaria," Journal of Infectious Diseases, vol. 178, no. 2, pp. 520$525,1998$.

[25] N. P. J. Day, T. T. Hien, T. Schollaardt et al., "The prognostic and pathophysiologic role of pro- and antiinflammatory cytokines in severe malaria," Journal of Infectious Diseases, vol. 180, no. 4, pp. 1288-1297, 1999.

[26] G. Biemba, V. R. Gordeuk, P. Thuma, and G. Weiss, "Markers of inflammation in children with severe malarial anaemia," Tropical Medicine and International Health, vol. 5, no. 4, pp. 256-262, 2000.

[27] M. N. Akhtar, S. Jamil, S. I. Amjad, A. R. Butt, and M. Farooq, "Association of malaria with thrombocytopenia," Annals of King Edward Medical College, vol. 11, pp. 536-537, 2005.

[28] Z. U. Rehman, M. Alam, A. Mahmood, A. Mubarik, A. Sattar, and K. A. Karamat, "Thrombocytopenia in acute malarial infection," Pakistan Journal of Pathology, vol. 10, pp. 9-11, 1999.

[29] E. M. Essien and M. I. Ebhota, "Platelet hypersensitivity in acute malaria (Plasmodium falciparum) infection in man," Thrombosis and Haemostasis, vol. 46, no. 2, pp. 547-549, 1981.

[30] R. D. Horstmann, M. Dietrich, U. Bienzle, and H. Rasche, "Malaria-induced thrombocytopenia," Blut, vol. 42, no. 3, pp. 157-164, 1981. 
[31] D. Mohanty, K. Ghosh, S. K. Nandwani et al., "Fibrinolysis, inhibitors of blood coagulation, and monocyte derived coagulant activity in acute malaria," American Journal of Hematology, vol. 54, no. 1, pp. 23-29, 1997.

[32] P. L. Olliaro and W. R. J. Taylor, "Developing artemisinin based drug combinations for the treatment of drug resistant falciparum malaria: a review," Journal of Postgraduate Medicine, vol. 50, no. 1, pp. 40-44, 2004.

[33] M. Adjuik, P. Babiker, P. Garner, P. Olliaro, W. Taylor, and N. White, "Artesunate combinations for treatment of malaria: meta-analysis," Lancet, vol. 363, no. 9402, pp. 9-17, 2004.

[34] F. Nosten and N. J. White, "Artemisinin-based combination treatment of falciparum malaria," The American Journal of Tropical Medicine and Hygiene, vol. 77, no. 6, pp. 181-192, 2007.

[35] N. J. White, "Assessment of the pharmacodynamic properties of antimalarial drugs in vivo," Antimicrobial Agents and Chemotherapy, vol. 41, no. 7, pp. 1413-1422, 1997.

[36] D. Bunnag, C. Viravan, S. Looareesuwan, J. Karbwang, and T. Harinasuta, "Double blind randomised clinical trial of oral artesunate at once or twice daily dose in falciparum malaria," Southeast Asian Journal of Tropical Medicine and Public Health, vol. 22, no. 4, pp. 539-543, 1991.

[37] C. Luxemburger, F. O. Ter Kulle, F. Nosten et al., "Single day mefloquin-artesunate combination in the treatment of multidrug resistant falciparum malaria," Transactions of the Royal Society of Tropical Medicine and Hygiene, vol. 88, no. 2, pp. 213217, 1994.

[38] O. P. Asthana, J. S. Srivastava, V. P. Kamboj et al., "A multicentric study with arteether in patients of uncomplicated Plasmodium falciparum malaria," Journal of Association of Physicians of India, vol. 49, pp. 692-696, 2001.

[39] N. Sethi, R. Srivastava, R. K. Singh, and P. S. R. Murthy, "Systamic toxicity study of a new schizontocidal antimalarial drug arteether in rats and monkeys," Indian Journal of Parasitology, vol. 12, no. 2, pp. 223-235, 1998.

[40] WHO, "Susceptibility of Plasmodium falciparum to antimalarial drugs. Report on global monitoring 1996-2004," WHO, Geneva, Switzerland, 2005.

[41] C. Falade, M. Makanga, Z. Premji, C.-E. Ortmann, M. Stockmeyer, and P. Ibarra de Palacios, "Efficacy and safety of artemether-lumefantrine (Coartem) tablets (six-dose regimen) in African infants and children with acute, uncomplicated falciparum malaria," Transactions of the Royal Society of Tropical Medicine and Hygiene, vol. 99, no. 6, pp. 459-467, 2005.

[42] J.-P. Guthmann, J. Ampuero, F. Fortes et al., "Antimalarial efficacy of chloroquine, amodiaquine, sulfadoxinepyrimethamine, and the combinations of amodiaquine + artesunate and sulfadoxine-pyrimethamine + artesunate in Huambo and Bié provinces, central Angola," Transactions of the Royal Society of Tropical Medicine and Hygiene, vol. 99, no. 7, pp. 485-492, 2005.

[43] P. Piola, C. Fogg, F. Bajunirwe et al., "Supervised versus unsupervised intake of six-dose artemether-lumefantrine for treatment of acute, uncomplicated Plasmodium falciparum malaria in Mbarara, Uganda: a randomised trial," Lancet, vol. 365, no. 9469, pp. 1467-1473, 2005.

[44] WHO, Guidelines for the Treatment of Malaria, World Health Organization, Geneva, Switzerland, 2006.

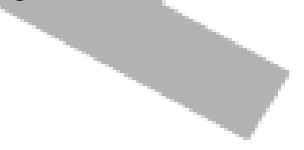

